\title{
The Effect of BML-111 in Preeclampsia Rat Model Induced by the Low Dose of Cadmium Chloride
}

\author{
KeKe Zhang ${ }^{1}$ Min Hu${ }^{1} \quad$ Lin Zhang ${ }^{1}$ \\ ${ }^{1}$ Department of Gynaecology and Obstetrics, JinHua Hospital of \\ Zhejiang University, Jinhua, Zhejiang, China \\ 2 Department of Gynaecology and Obstetrics, The Second Affiliated \\ Hospital of Wenzhou University, WenZhou, Zhejiang, China \\ ${ }^{3}$ Department of Gynaecology and Obstetrics, The First Affiliated \\ Hospital of Wenzhou University, WenZhou, Zhejiang, China
}

Yinping Huang ${ }^{3}$

\author{
Address for correspondence Yinping Huang, MD, Department of \\ Gynaecology and Obstetrics, The First Affiliated Hospital of Wenzhou \\ University, WenZhou, 325000, Zhejiang, China \\ (e-mail: 1336476266@qq.com).
}

Am J Perinatol Rep 2019;9:e201-e208.

\begin{abstract}
Keywords

- preeclampsia

- BML-111

- cadmium chloride

- inflammation

Aim This article determines the optimal time and dose of cadmium chloride $\left(\mathrm{CdCl}_{2}\right)$ injected to pregnant rat to establish experimental preeclampsia (PE) model. In addition, the therapeutic potential of BML-111, a lipoxin $\mathrm{A} 4$ analogue, in the $\mathrm{CdCl}_{2}$-induced $\mathrm{PE}$ model was also evaluated.

Methods Peritoneal injection of two dose of $\mathrm{CdCl}_{2}$ for successive 6 days was tested in the pregnant rats starting from various gestational days (GDs). During this process, the systolic blood pressure and the body weight of pregnant rats and neonatal rats were monitored. The pathological changes of the placenta and kidney were evaluated by hematoxylin and eosin staining. The phosphorylation of extracellular signal-regulated kinase $1 / 2$ and signal transducer and activator of transcription 3 in the placentas was detected by Western blot, and the messenger ribonucleic acid expression of interleukin (IL)-6, tumor necrosis factor- $\alpha$, and IL-10 in the placentas were detected by real-time polymerase chain reaction. BML-111 at the dose of $1 \mathrm{mg} / \mathrm{kg} /$ day was peritoneally injected into the rat after establishing the PE model to test its therapeutic potential. Results In the present study, we successfully established the PE model in pregnant rats by intraperitoneally injection of $\mathrm{CdCl}_{2}$ at the dose of $0.125 \mathrm{mg} / \mathrm{kg} /$ day from GD 9 to 14 . We recapitulated multiple features of clinical $\mathrm{PE}$ in $\mathrm{CdCl}_{2}$-induced rat, including high blood pressure, renal dysfunction, and inflammatory response in placenta. Furthermore, treatment with BML-111 significantly relieved multiple features in our PE rat model.

Conclusions BML-111 has a potential therapeutic effect in pregnant rats with $\mathrm{CdCl}_{2}$ induced PE, which appears to be mediated through inhibition of inflammatory processes in the placenta.
\end{abstract}

Preeclampsia (PE) is a multisystem disorder that generally appears after 20 weeks of gestation and is characterized by hypertension, proteinuria, and edema, and can be accompanied by multiple organ dysfunction including heart, liver, kidney, and brain. ${ }^{1}$ PE affects approximately 2 to $8 \%$ of pregnancies worldwide, ${ }^{2}$ and this disorder is one of the important causes for maternal, fetal, and neonatal mortality

received

December 23, 2018 accepted after revision May 16, 2019
DOI https://doi.org/

10.1055/s-0039-1693016. ISSN 2157-6998. and morbidity. Presently, delivery of the fetus and placenta remains the only cure for PE. Therefore, new therapeutic strategy is urgently needed.

The exact etiology of PE is complex and the underlying molecular mechanisms remain elusive. Recently, "excessive inflammatory response" was regarded as the main cause of PE. The evidence supporting this inflammatory perspective
Copyright $\odot 2019$ by Thieme Medical Publishers, Inc., 333 Seventh Avenue, New York, NY 10001, USA. Tel: +1(212) 584-4662.
License terms

(요 (1) $\Theta$ 
came from the observation that inflammatory mediators such as interleukin (IL), tumor necrosis factor $\alpha$ (TNF- $\alpha$ ), C-reactive protein (CRP), and serum amyloid P-like material in the serum of PE patients were significantly higher than those in normal pregnant women. ${ }^{3}$ Several studies have suggested that a high concentration of circulating TNF- $\alpha$ is associated with more severe clinical manifestations of PE. ${ }^{4}$

With the rapid development of global industrialization, the influence of chemical substances cadmium $(\mathrm{Cd})$ to humans and animals is also growing.,6 Currently, $\mathrm{Cd}$ has been regarded as the seventh toxic substance that is harmful to human health by both the Agency for Toxic Substances and Disease Registry and the Environmental Protection Agency. Exposure for Cd can be obtained through several ways either by food intake, cigarette smoke, ${ }^{7}$ or emissions from industrial activities and waste management operations. ${ }^{8}$ Pregnant women are more vulnerable to $\mathrm{Cd}$ because of the greatly increased absorption and retention of $\mathrm{Cd}$ caused by nutritional deficiencies during pregnancy. ${ }^{9} \mathrm{Cd}$ can cause damage in multiple organs including the placenta. $\mathrm{Cd}$ has been linked with many human diseases, such as cancer, bone disease, ${ }^{10}$ and hypertension. Kolusari et al reported that the level of serum Cd significantly increased in women suffering from $\mathrm{PE} .^{11}$ The causal role of $\mathrm{Cd}$ in hypertensive disorders has been documented in an animal study, in which the successful replication of the toxemia model can be done by injecting pregnant rats with the $\mathrm{Cd}$ solution. ${ }^{12}$

Recently, many studies showed a close relationship between $\mathrm{Cd}$ and PE. ${ }^{13,14}$ The toxic effects of $\mathrm{Cd}$ share similar features with the clinical manifestations of PE, such as hypertension, proteinuria, decreased placental perfusion, fetal growth restriction, convulsions, and dysfunction of liver and kidney. ${ }^{15}$ Although the placenta has a certain barrier to $\mathrm{Cd}$, it is still an important target organ for $\mathrm{Cd}$. Cd exposure during pregnancy may lead to the occurrence of PE by changing the structure of the placenta and the function of placental transfer. Low dose of Cd was reported to promote the secretion of IL-6, TNF- $\alpha$, and other inflammatory substances in a variety of cells. In addition, a considerable number of studies also reported that $\mathrm{Cd}$ exposure could lead to oxidative stress. As PE in pregnancy is characterized by systemic inflammatory response, oxidative stress, vascular endothelial cell injury, and dysfunction, it is reasonable that experimental PE model could be established by injecting low dose of $\mathrm{Cd}$, and such a model can be used to evaluate new therapeutic strategies in PE.

Lipoxin A4 (LXA4) is a biologically active eicosanoid that is present at the inflammatory sites and appears to be an endogenous anti-inflammatory mediator. ${ }^{16}$ Several in vitro and in vivo studies have revealed that LXA4 function as innate "stop signals," acting to control local inflammatory processes. ${ }^{17}$ After binding with its receptor, LXA4 plays an important role in inflammation, oxidative stress, and immune regulation. LXA4 is rapidly converted to inactive lipids with lower potencies as anti-inflammatory agents. To circumvent such degradation, stable LXA4 analogues has been designed. ${ }^{16}$ BML-111 is a C-7 truncated analogue that was originally proven to be equiactive with LXA4 in the inhibition of leukotriene B4-induced neutrophil chemotaxis.
Recent study found that LXA4 receptor is abundantly expressed in the placenta, endometrium, and muscle layer. Many studies demonstrated a beneficial role of LXA4 in treating hypertensive disorders which occur during pregnancy. LXA4 can not only inhibit the release of inflammatory cytokines such as TNF- $\alpha$, IL-1, IL-6, IL-8, and IL-13, but also can weaken the ability of neutrophils for forcing and adhering during pregnancy. In addition, LXA4 can also downregulate the secretion of Th1 cytokines and upregulate Th2 cytokines secretion, favoring the anti-inflammatory effects.

In this study, we replicate a rat PE model by intraperitoneally injection of $\mathrm{Cd}$ and explored whether the analogue of LXA4 BML-111 has the therapeutic effect.

\section{Material and Method}

\section{Animals}

Adult Wistar female rats, 8 to 10 weeks old and weighted at 200 to $220 \mathrm{~g}$, were purchased from the experimental animal center of Weitong Lihua (Beijing, China) for studies approved by the Animal Care and Use Committee of Tongji Medical College. All animals were housed individually in cages under pathogenfree conditions with controlled temperature at $20^{\circ} \mathrm{C}$ to $25^{\circ} \mathrm{C}$ and a 12-hour light and dark cycle. All the rats were fed with a standard laboratory diet and water ad libitum. The female rats with normal basic systolic blood pressure (SBP) were mated with fertile male rats overnight. The next day was defined as day 1 of gestation (GD 1) if a vaginal plug is visible or sperms detected in vaginal smear.

\section{Confirm the Suitable Time and Dose for Replicating Rat PE Model}

Twenty-five Wistar pregnant rats were randomly divided into five groups $(n=5)$ : control group, D7 group, D9 group, D11 group, and D13 group and were intraperitoneally injected with Cd chloride $\left(\mathrm{CdCl}_{2}\right)$ (Sinopharm Chemical Reagent Co. Ltd.) at $0.125 \mathrm{mg} / \mathrm{kg} /$ day, which was dissolved in sterile saline, respectively, beginning from GD 7, 9, 11, and 13 for 6 days, and the rats of the control group were intraperitoneally injected with sterile saline. The differences in blood pressure among the groups were compared which confirm the suitable time for establishing the PE model. After that, another 32 pregnant Wistar rats were randomly divided into three groups $(n=8)$ : the control group, the $0.25 \mathrm{mg} / \mathrm{kg} /$ day $\mathrm{CdCl}_{2}$ group, $0.125 \mathrm{mg} / \mathrm{kg} /$ day $\mathrm{CdCl}_{2}$ group, and the $0.0625 \mathrm{mg} / \mathrm{kg} / \mathrm{day} \mathrm{CdCl}_{2}$ group to determine the lowest but effective dose. The control group only received sterile saline. The blood pressure and maternal weight of the pregnant rats were monitored throughout the pregnancy period.

\section{To Explore the Therapeutic Effect of the Analogue of Lipoxid Acid (BML-111) in the Rat PE Model Induced by Low Dose of $\mathbf{C d C l}_{2}$}

We use intraperitoneally injected with $\mathrm{CdCl}_{2} 0.125 \mathrm{mg} / \mathrm{kg} /$ day from gestation day 9 to 14 for 6 days to establish the rat PE model. Twenty-four Wistar pregnant rats were randomly divided into three groups $(n=8)$ : the control group, Cd group, and $\mathrm{Cd}+\mathrm{BML}-111$ group, and were respectively 
intraperitoneally injected with sterile saline, $0.125 \mathrm{mg} / \mathrm{kg} /$ day $\mathrm{CdCl}_{2}, 0.125 \mathrm{mg} / \mathrm{kg} / \mathrm{day} \mathrm{CdCl}_{2}$, and $1 \mathrm{mg} / \mathrm{kg} /$ day BML-111 for 6 days.

\section{Measurement of Systolic Blood Pressure}

The SBPs were measured in conscious, restrained pregnant rats in the morning on GD 0,9,13,16, and 19. An automated system with a photoelectric sensor linked to a dual channel recorder (BP-98A, Softron, Japan), tail cuff, and sphygmomanometer was used to obtain indirect SBP measurement. On day 20 of gestation, the rats were anesthetized and fetal pups were delivered by caesarean section. The fetal pups were weighed. The kidney and placentas were stored at $-80^{\circ} \mathrm{C}$ for further assessment.

\section{Determination of Urinary Albumin Excretion}

On day 3 and 19 of pregnancy, the rats were placed in metabolic cages to collect 24-hour urine. To avoid contaminating the collected urine, rats were allowed free access to water but were restricted from food. To avoid the adverse effects of fasting, rats were fed in other cages for 30 minutes every 6 hours. Urinary protein concentrations were determined with a bicinchoninic acid (BCA) protein assay kit (Thermo Fisher Scientific, Rockford, IL) using bovine serum albumin (BSA) as the standard.

\section{Western Blot Analysis}

Protein concentrations were determined using a BCA protein assay kit (Thermo Fisher Scientific). Then, we separated the equal amount of sample proteins $(40 \mu \mathrm{g})$ on a $10 \%$ sodium dodecyl sulfate-polyacrylamide gel electrophoresis, and transferred them onto polyvinylidene difluoride membranes (Millipore, Billerica, MA). The membranes were blocked in Tris-buffered saline with $0.1 \%$ Tween 20 (TBST) which contained 5\% BSA for 1 hour and incubated with specific primary antibodies, including anti-extracellular signal-regulated kinase 1/2 (ERK1/2) (1:1000; CST, Danvers, MA), anti-pERK1/2 (1:1000; CST), anti-signal transducer and activator of transcription 3 (STAT3) (1:1000; CST), anti-p-STAT3 (1:2000; CST), and anti- $\beta$-actin (1:1000; CST) overnight at $4^{\circ} \mathrm{C}$. The membranes were washed three times in TBST and then incubated with horseradish peroxidase-conjugated secondary antibodies (1:10000) for 1 hour at room temperature. Protein bands were visualized by an enhanced chemiluminescent kit (Millipore, Bedford, MA) and densitometric analysis was performed using Image $\mathrm{J} 1.47$. The $\beta$-actin served as an internal control.

\section{Quantitative Real-Time Reverse Transcription- Polymerase Chain Reaction}

The placentas were lysed with TRizol reagent (Invitrogen, Carlsbad, CA), and the total ribonucleic acid (RNA) was extracted according to the manufacturer's instructions. For real-time reverse transcription-polymerase chain reaction (RT-PCR) assays, the complementary deoxyribonucleic acid (CDNA) sequences of all detected genes were retrieved from the National Center for Biotechnology Information database. The primers were designed with the Oligo Primer Analysis
Table 1 The sequences of primers used in real-time PCR

\begin{tabular}{|l|l|l|}
\hline $\begin{array}{l}\text { Target } \\
\text { gene }\end{array}$ & Prime & Sequence $\left(5^{\prime}-\mathbf{3}^{\prime}\right)$ \\
\hline \multirow{2}{*}{ IL-6 } & Forward & ATA TGT TCT CAG GGA GAT CTT GGA A \\
\cline { 2 - 3 } & Reverse & GTG CT CT CGC TGT TCA TAC A \\
\hline \multirow{2}{*}{ IL-10 } & Forward & GTA GAA GTG ATG CCC CAG GC \\
\cline { 2 - 3 } & Reverse & AGA AAT CGA TGA CAG CGT CG \\
\hline \multirow{2}{*}{ TNF- $\alpha$} & Forward & ACT GAA CTT CGG GGT GAT CG \\
\cline { 2 - 3 } & Reverse & TGG TGG TTT GCT ACG ACG TG \\
\hline \multirow{2}{*}{ GAPDH } & Forward & GGC ACA GTC AAG GCT GAG AAT G \\
\cline { 2 - 3 } & Reverse & ATG GTG GTG AAG ACG CCA GTA \\
\hline
\end{tabular}

Abbreviations: GAPDH, glyceraldehyde 3-phosphate dehydrogenase; IL, interleukin; TNF, tumor necrosis factor.

4.0 software (Molecular Biology Insights, Cascade, $\mathrm{CO}$ ), and the sequences were subjected to Basic Local Alignment Search Tool analysis. The total RNA $(2 \mu \mathrm{g})$ was used for reverse transcription using ReverTra ACE (TOYOBO, Osaka, Japan) in a volume of $20 \mathrm{~mL}$. Next, $1.5 \mu \mathrm{L}$ cDNA was amplified with Thunderbird SYBR qPCR Mix (TOYOBO) in duplicate. The resulting data were analyzed with the comparative cycle threshold method for relative gene expression quantification against glyceraldehyde 3-phosphate dehydrogenase. The primers for all genes tested are listed in - Table $\mathbf{1}$.

\section{Histology Assay}

Placentae and kidneys specimens in $4 \mu \mathrm{m}$ paraffin sections were stained with hematoxylin and eosin for conventional morphological evaluation under light microscope (Olympus BX51, Tokyo, Japan).

\section{Statistical Analysis}

Data were presented as mean \pm standard error of the mean from at least three independent experiments performed in triplicate. Differences between two means were determined using Student's $t$-test. One-way analysis of variance was performed for the comparison of multiple groups. A $p$-value of less than 0.05 was considered significant.

\section{Results}

\section{The Suitable Time and Dose of $\mathrm{CdCl}_{2}$ to Establish Rat PE Model}

After intraperitoneally injected with $0.125 \mathrm{mg} / \mathrm{kg} / \mathrm{day} \mathrm{CdCl}_{2}$, the SBPs of the D9 group increased steadily and the difference was statistically significant. In contrast, the SBP of other groups including the D7 group, D11 group, and D13 group increased slightly and no significant changes were observed. These results suggested that the suitable time to establish a PE model in rat can be done by injection with $0.125 \mathrm{mg} / \mathrm{kg} /$ day $\mathrm{CdCl}_{2}$ at gestation day 9 to 14 (-Fig. 1A). In addition, we also tested the effects of different doses of $\mathrm{CdCl}_{2}$ on the blood pressure, and found that the $0.125 \mathrm{mg} / \mathrm{kg} /$ day $\mathrm{CdCl}_{2}$ group and the $0.25 \mathrm{mg} / \mathrm{kg} / \mathrm{day} \mathrm{CdCl}_{2}$ group all had higher SBP 

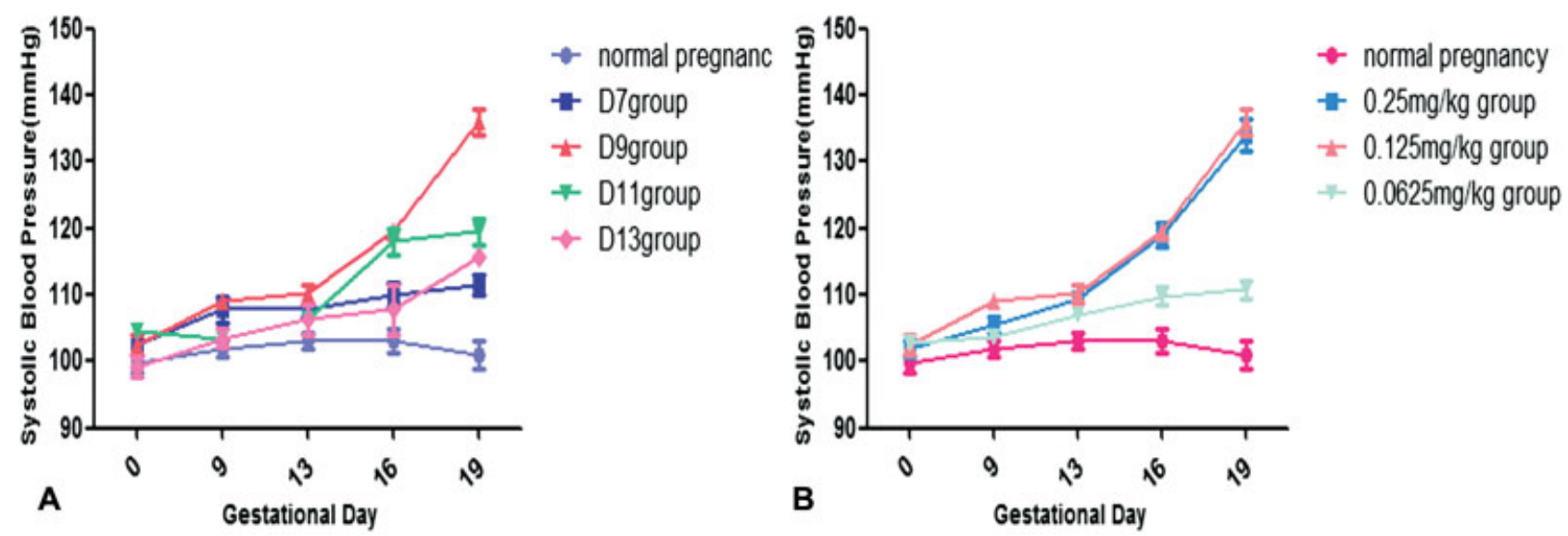

Fig. 1 Pregnant Wistar rats develop preeclampsia-like symptoms in response to cadmium chloride $\left(\mathrm{CdCl}_{2}\right)$. Pregnant Wistar rats were treated with $0.125 \mathrm{mg} / \mathrm{kg} /$ day $\mathrm{CdCl}_{2}$ from gestational day $7,9,11$, and 13 for 6 days, systolic blood pressure is presented in (A). Pregnant Wistar rats were given saline or $0.0625,0.125$, and $0.25 \mathrm{mg} / \mathrm{kg} /$ day $\mathrm{CdCl}_{2}$ from gestational day 9 to 14 , systolic blood pressure is presented in (B).

(-Fig. 1B). Taken together, $\mathrm{CdCl}_{2}$ at $0.125 \mathrm{mg} / \mathrm{kg} /$ day was chosen as the lowest but effective dose to establish a rat PE model.

\section{BML-111 Relieved the Phenotype in PE Model Rat}

After we established the PE model by intraperitoneal injection with $\mathrm{CdCl}_{2} 0.125 \mathrm{mg} / \mathrm{kg}$ from gestation day 9 to 14 in pregnant rat, we then tested the protective effects of BML-111
$1 \mathrm{mg} / \mathrm{kg} /$ day for consecutively 6 days from gestation day 14 . We found that BML-111 significantly alleviated the SBP in PE model ( - Fig. 2A). In addition, we also monitored the body weight of maternal during pregnancy and the neonatal rats after giving birth. Reduced maternal body weight gain was also observed in the Cd group compared with that of the BML-111 administration group $(94.33 \pm 4.14 \mathrm{~g}$ vs. $111.33 \pm 3.09 \mathrm{~g}, \mathrm{Cd}$ + BML-111 group vs. Cd group, $p<0.05$, - Fig. 2B). However,
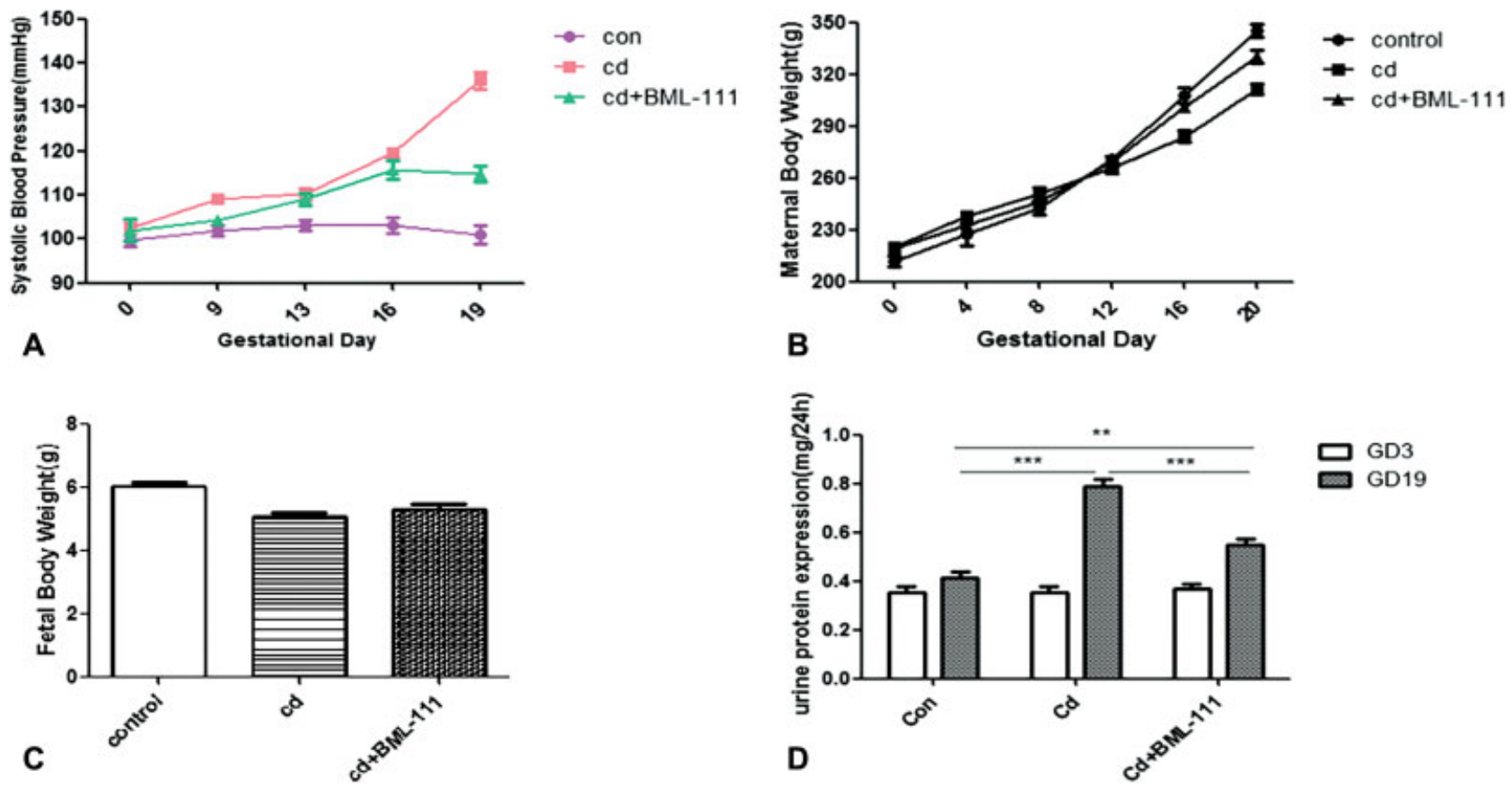

Fig. 2 BML-111 treatment improves the symptoms of preeclampsia (PE) model rats. Pregnant rats were intraperitoneally injected with sterile saline (control group) or $0.125 \mathrm{mg} / \mathrm{kg}$ cadmium chloride $\left(\mathrm{CdCl}_{2}\right)$ (Cd group) on day 9 to 14 of pregnancy. BML-111 (Cd + BML-111 group, $1 \mathrm{mg} / \mathrm{kg} /$ day) was administrated intraperitoneally injected after $\mathrm{Cd}$ administration from day 14 of pregnancy and at the rate of once per day for 6 days. (A) The systolic blood pressures (SBPs) of the Cd group at days 13,16 , and 19 of pregnancy were $110.3 \pm 2.06,119.5 \pm 2.1$, and $136 \pm 3.39 \mathrm{~mm} \mathrm{Hg}$, respectively $(n=8, p<0.05$, vs. the control group at the corresponding time point). BML-111 treatment significantly alleviated SBP to $114.8 \pm 2.95 \mathrm{~mm} \mathrm{Hg}$ on days 19 of pregnancy, respectively ( $n=8, p<0.05$, vs. the Cd group). (B) The maternal body weight gain of the Cd group ( $94.33 \pm 4.14 \mathrm{~g}$ ) was significantly lower compared with that of the BML-111 administration group (111.33 $\pm 3.09 \mathrm{~g}, n=8, p<0.05)$. (C) The fetal weight of the Cd group was not apparently different compared with that of the placebo and BML-111 administration groups $(p>0.05)$. (D) Twenty-four-hour urinary protein excretions on days 3 and 19 of pregnancy were presented. There were no difference among the three groups on day 3 of pregnancy ( $p>0.05)$, but on day 19 of pregnancy, after BML-111 administration, this level $\left(0.41 \pm 0.06 \mathrm{mg} / 24\right.$ hours) was significantly lower than in the Cd group $\left(0.79 \pm 0.07 \mathrm{mg} / 24\right.$ hours, $\left.n=8,{ }^{* * *} p<0.001\right)$. All data are expressed as the mean \pm standard error of the mean (SEM). ${ }^{*} p<0.05,{ }^{* *} p<0.01,{ }^{* * *} p<0.001$. 
no apparent difference was observed in the neonatal body

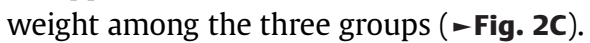

We also evaluated renal function by detection of 24-hour urinary albumin excretion. On day 3 of gestation, before intraperitoneally injected with $\mathrm{CdCl}_{2}$ or sterile saline solution, mean urinary albumin excretion of pregnant rats showed no difference between the control group and model group. However, on day 19 of gestation, the level of urinary albumin was significantly higher in the $\mathrm{Cd}$ group $(0.79 \pm 0.07$ vs. $0.41 \pm 0.06 \mathrm{mg} / 24 \mathrm{~h}, p<0.001)$. Interestingly, the level of urinary albumin was notably decreased by treatment with BML-111 in the PE model $(0.55 \pm 0.06$ vs. $0.79 \pm 0.07 \mathrm{mg} / 24 \mathrm{~h}, p<0.001$ ) (-Fig. 2D).

\section{BML-111 Inhibited the Phosphorylation Level of ERK 1/2 and STAT3}

ERK and STAT are key players in mediating inflammation. We then examined the effect of BML-111 on these two signaling pathways. As shown in - Fig. 3A-D, activation of ERK and STA3 were observed in the PE model group induced by $\mathrm{CdCl}_{2}$, evidenced by the increment level of p-ERK1/2 and p-STAT3 expression, while both of them were reversed by BML-111 treatment.

\section{BML-111 Inhibited IL-6, TNF- $\alpha$ Generation, and Enhanced IL-10 Expression in PE Rats}

IL-6 and TNF- $\alpha$ were important proinflammatory cytokines that participated in the pathogenesis of PE. In this study, we found that the messenger RNA (mRNA) level of IL-6 and
TNF- $\alpha$ was increased in the PE group that was induced by $\mathrm{Cd}$ but decreased after BML-111 treatment. In addition, an antiinflammatory factor, IL-10 mRNA expression was also decreased in the $\mathrm{Cd}$ group and recovered in the treatment group by BML-111 ( $p<0.05)$ (-Fig. 4A-C).

\section{BML-111 Alleviated Morphological Changes in the Placenta and Kidney}

In this study, intraperitoneally injecting with $\mathrm{CdCl}_{2}$ caused morphological changes in both the placenta and kidney characterized by formation of microthrombosis and glomerular endotheliosis with accumulation of inflammatory leukocytes. But these changes were remarkably relived by treatment with BML-111 (shown in -Fig. 5A-F).

\section{Discussion}

In the past decades, researchers continuously tried to establish animal PE models by various approaches, such as uteroplacental ischemia, drug-induced vasoconstriction, placenta thrombosis simulation, and placental ischemia and hypoxia. However, none of these animal models could recapitulate most of the clinical symptoms in human PE exactly. Therefore, establishing a suitable experimental PE model is important for both mechanistical investigation and for therapeutic agent evaluation. In the present study, we presented a new way to establish the experimental PE model by intraperitoneal injection of low dose $\mathrm{CdCl}_{2}$ in pregnant rat from gestation day 9 to 14 . Such a model recapitulated most complications in human PE.
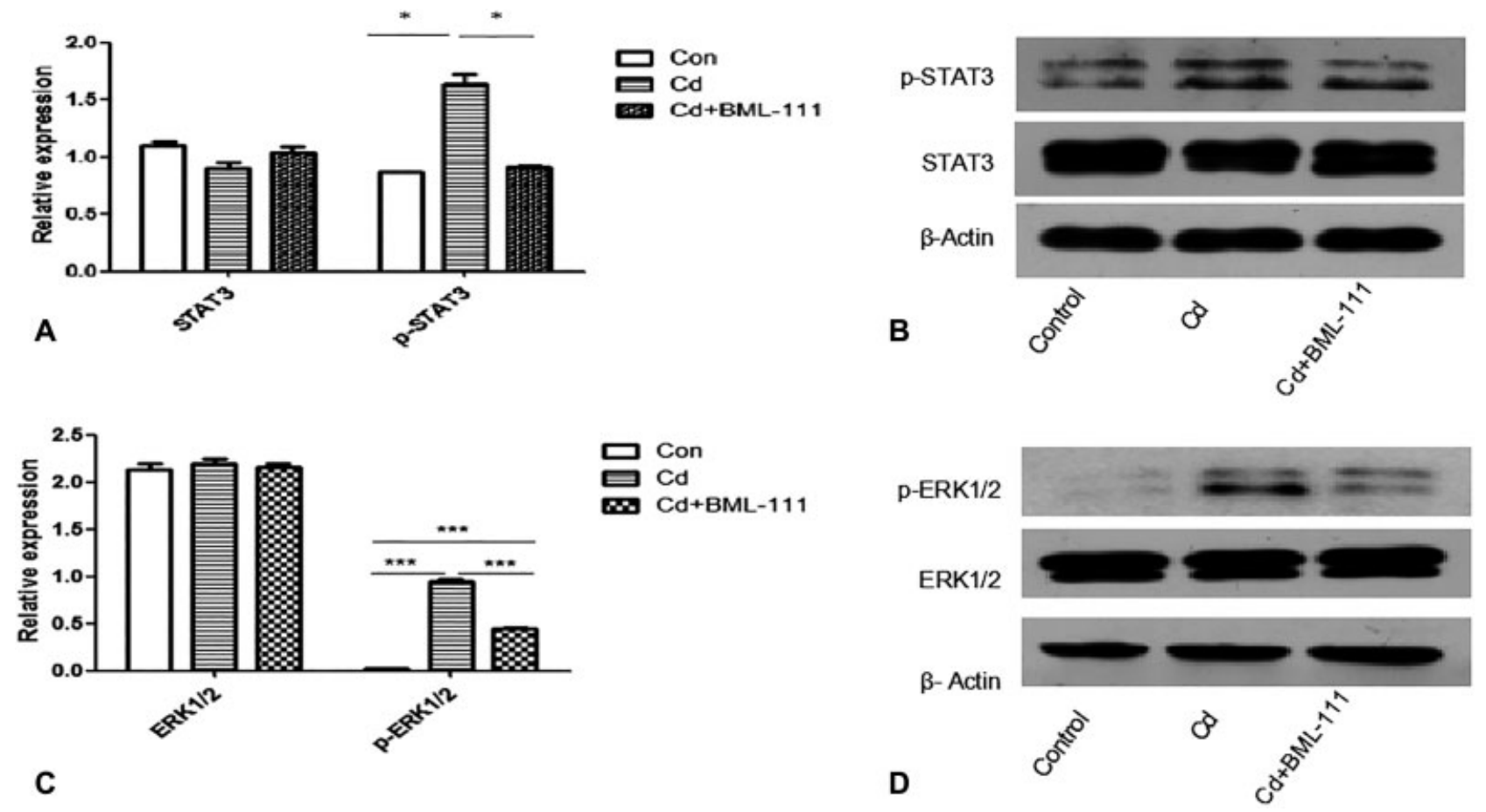

Fig. 3 BML-111 inhibited the phosphorylation level of extracellular signal-regulated kinase 1/2 (ERK1/2) and signal transducer and activator of transcription 3 (STAT3). Assay the phosphorylation level of ERK1/2 and STAT3 in the placenta from the three groups. (A-D) The ERK1/2, p-ERK1/2, STAT3, and p-STAT3 protein expression in the placenta from the three groups were determined by Western blot analyses ( $\beta$-actin served as an internal control). Densitometric analysis of the visualized bands was performed using the Gel-Pro analyzer 4 software to normalize ERK1/2, p-ERK1/2, STAT3, and p-STAT3 protein expression with the corresponding $\beta$-actin levels. Results were expressed as mean \pm standard error of the mean (SEM) $(n=8, p<0.05)$. The data shown are representative of three independent experiments. 

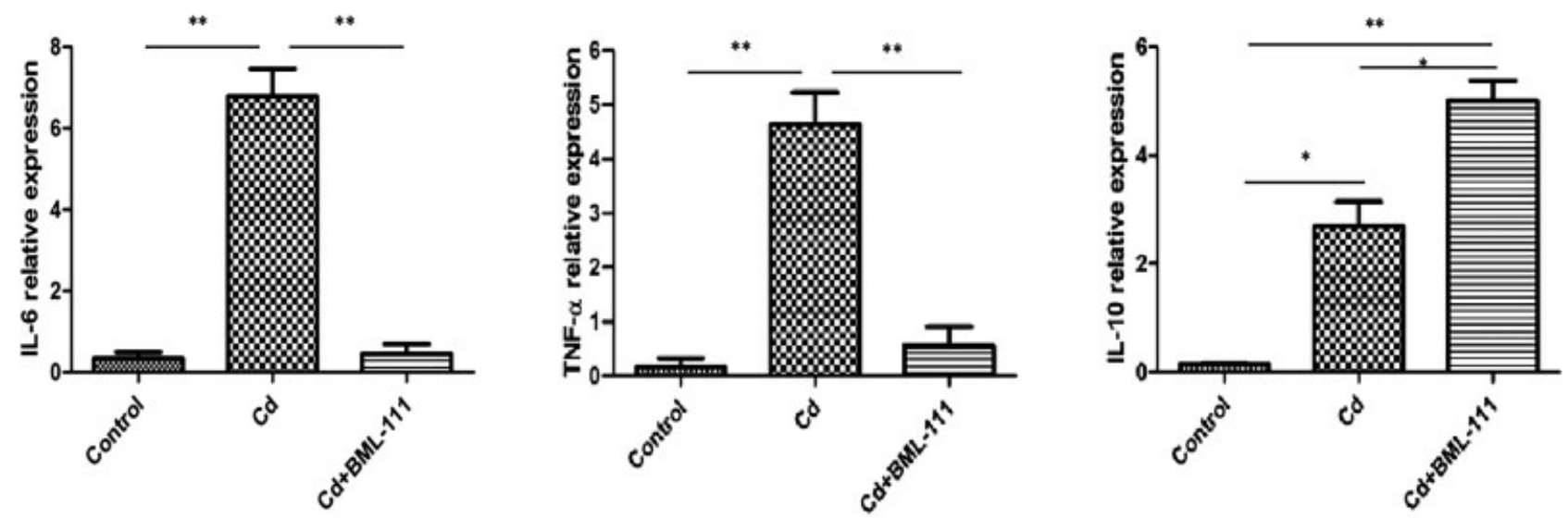

Fig. 4 BML-111 downregulates interleukin (IL)-6 and tumor necrosis factor (TNF)- $\alpha$, while upregulates IL-10. (A-C) Reverse transcription-polymerase chain reaction (RT-PCR) analyses of IL-6, TNF- $\alpha$, and IL-10 messenger ribonucleic acid (mRNA) expression in the placenta from the three groups (glyceraldehyde 3-phosphate dehydrogenase [GAPDH] severed as an internal control). Cadmium (Cd) enhanced the expression of IL- 6 and TNF- $\alpha$ mRNA compared with the control group, while inhibited IL-10 mRNA expression $\left(n=8,{ }^{* *} p<0.01,{ }^{*} p<0.05\right)$. And the mRNA expression of IL-10 was upregulated in the placenta of the Cd + BML-111 group, while IL-6 and TNF- $\alpha$ were downregulated $\left(n=8,{ }^{* *} p<0.01,{ }^{*} p<0.05\right)$.

\section{Control Group}

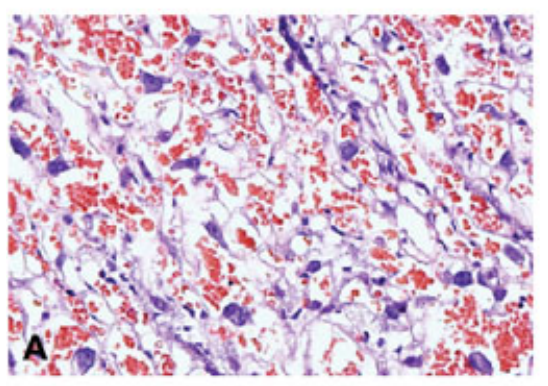

\section{Control Group}

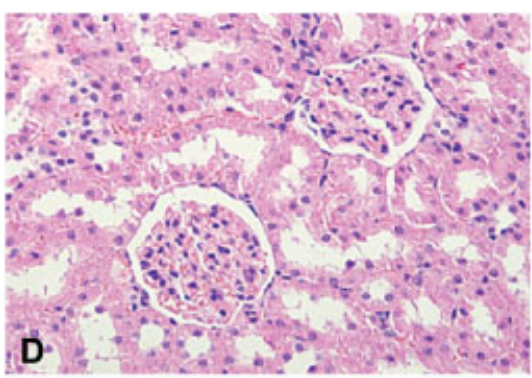

Cd Group

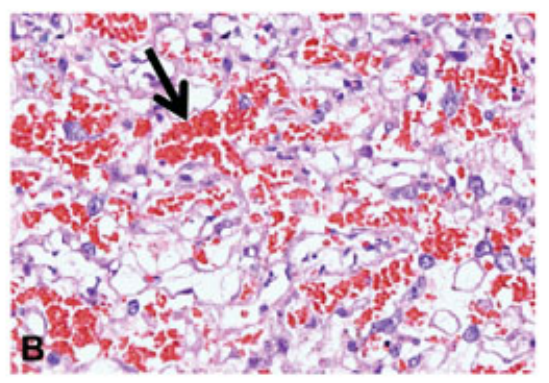

Cd Group

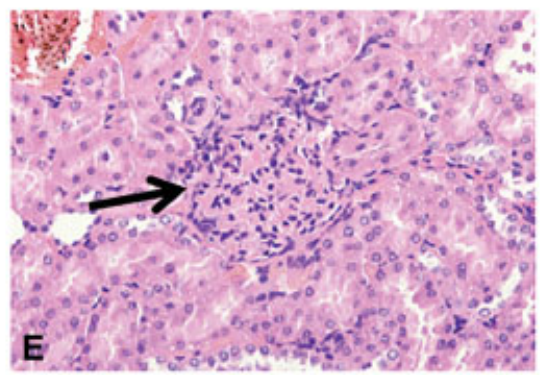

\section{Cd+BML-111 Group}

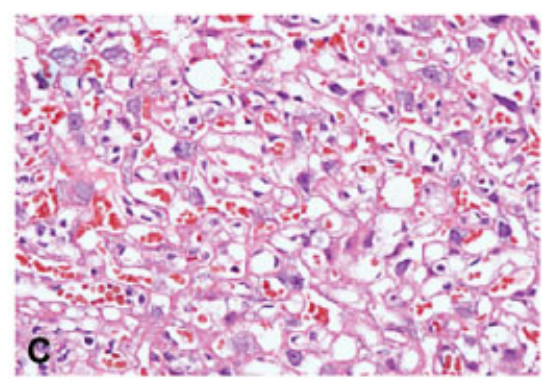

\section{Cd+BML-111 Group}

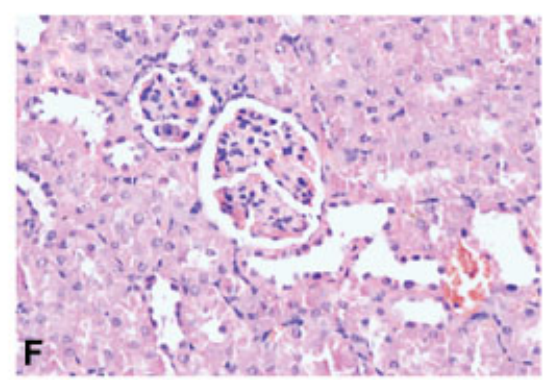

Fig. 5 Hematoxylin and eosin stain for placental and kidney pathological analysis. Placenta and kidney specimens were obtained from normal pregnancy rats, $0.125 \mathrm{mg} / \mathrm{kg} /$ day cadmium (Cd)-treated rats, and $0.125 \mathrm{mg} / \mathrm{kg} /$ day Cd $+1 \mathrm{mg} / \mathrm{kg} /$ day BML- 111 administration rats on day 20 of pregnancy. $(A-C)$ Representative images of the placenta (original magnification $\times 200$ ). The black arrow indicates the degeneration in the placental labyrinth. (D-F) Representative images of the placenta (original magnification $\times 400$ ). Black arrow indicates reduced capillary space in the kidney.

As one of the toxic elements and environmental pollutants, Cd was reported to have a relationship with human PE. Many previous studies have demonstrated that a higher level of $\mathrm{Cd}$ is present in the peripheral blood of preeclamptic women than that of women undergoing normal pregnancies. ${ }^{13}$ The most conclusive evidence that links $\mathrm{Cd}$ with hypertensive disorders came from an animal experiment launched in 1965 by Henry, who successfully replicated the hypertensive model by injecting pregnant rats with the aqueous solution of $\mathrm{Cd} .{ }^{12}$ The toxic effects of Cd share strikingly similar features to many of clinical manifestations of PE, such as hypertension, proteinuria, and lesion of the liver and kidney. Previous studies have shown that exposure of pregnant rats to relatively low dose of $\mathrm{Cd}$ could induce hypertension, proteinuria, and other symptoms of PE. ${ }^{18}$ In consistent with this, in this study, we also found that administration of $\mathrm{CdCl}_{2}$ at a concentration of $0.125 \mathrm{mg} / \mathrm{kg} /$ day to pregnant rats from 
gestation day 9 to 14 increased blood pressure and induced proteinuria. Experimental and epidemiological studies showed that Cd could induce hypertension through several pathways such as oxidative stress, vasoconstriction, renal proximal tubular injury, sodium retention, and altered catecholamine metabolism. ${ }^{19,20}$ In addition, Cd also has a proinflammatory effect. Olszowski et al showed that low dose of $\mathrm{Cd}$ could induce the release of inflammatory factors, such as IL-1, IL-6, and CRP, in both in vivo and in vitro experiments. ${ }^{21}$

The kidneys are an important target of chronic $\mathrm{Cd}$ exposure. $^{22}$ During pregnancy, $\mathrm{Cd}$ is mobilized from the liver to the placenta and kidneys, ${ }^{23}$ and favors the movement of $\mathrm{Cd}$ to the kidneys. Therefore, kidneys are in great risk of experiencing Cd toxicity during pregnancy. Pathological changes in the maternal placenta and kidney of those seen in PE were also observed in the pregnant rats after $0.125 \mathrm{mg} / \mathrm{kg} /$ day of $\mathrm{CdCl}_{2}$ treatment. The kidneys of animals in the Cd group displayed swelling of endothelial cells that appeared to reduce the capillary space (glomerular endotheliosis). Cd administration also caused degeneration in the placental labyrinth.

As the most potent endogenous stop signals for inflammation, LXA4 plays an important role in the inflammatory response by binding to its receptor. However, LXA4 can be rapidly converted to inactive lipids in vivo with lower potencies. Currently, multiple LXA4 analogues with improved pharmacokinetics have been designed and showed potential anti-inflammatory activity. BML-111, a C-7 truncated LXA4 analogue, has been proved to be equiactive with LXA4 in the inhibition of leukotriene B4-induced neutrophil chemotaxis. In addition, BML-111also has therapeutic potential in classical PE model induced by lipopolysaccharides. In the present study, we further explored the benefit of BML-111 in our PE model induced by low dose of $\mathrm{CdCl}_{2}$. In the present study, we found that BML-111 significantly relieved various pathological phenotypes of PE mode induced by low dose $\mathrm{CdCl}_{2}$. These data suggest that BML-111 may be a potential candidate in PE treatment.

Inflammation has been implicated in the pathogenesis of PE. Inflammatory cytokines and activation inflammationrelated signal pathways such as ERK and STAT3 were shown to be upregulated in human PE. Szabo et al found that the level of ERK phosphorylation in PE patients was significantly higher than that of normal pregnant women in trophoblast cells. ${ }^{24}$ Sharma et al also found that serum levels of IL-6, IL-8, and TNF- $\alpha$ were all increased in PE patients, while IL-10, an anti-inflammatory cytokine, was decreased. ${ }^{25}$ Consistently, in the present study, we found that compared with normal pregnant group, the phosphorylation level of ERK and STAT3 and the expression level of IL- 6 and TNF- $\alpha$ were significantly elevated by $\mathrm{Cd}$ exposure during pregnancy, while decreased after treatment with BML-111. Therefore, our results further confirmed the important role of inflammation in PE pathogenesis and highlight the anti-inflammatory role of BML-111 in PE model that is induced by $\mathrm{Cd}$.

In summary, we identified a new way of establishing the PE model in rat by intraperitoneal injection of $\mathrm{CdCl}_{2}$ at
$0.125 \mathrm{mg} / \mathrm{kg} /$ day from gestation day 9 to 14 . Cd-mediated inflammation cytokines are key mechanisms underlying the features of $\mathrm{PE}$ induced by $\mathrm{CdCl}_{2}$ in pregnant rats. Our data demonstrate that BML-111 reduces the production of inflammation factors by reducing the expression of the phosphorylation level of ERK and STAT3. These findings showed novel therapeutic options for the management of this serious condition.

\section{Conflict of Interest}

None.

\section{References}

1 Redman CW, Sargent IL. Latest advances in understanding preeclampsia. Science 2005;308(5728):1592-1594

2 Steegers Ea P, Peter VD, Duvekot JJ, et al. Pre-eclampsia. Lancet 2010;376(9741):631-644

3 Szarka A, Rigó J Jr, Lázár L, Beko G, Molvarec A. Circulating cytokines, chemokines and adhesion molecules in normal pregnancy and preeclampsia determined by multiplex suspension array. BMC Immunol 2010;11(01):59

4 Udenze I, Amadi C, Awolola N, Makwe CC. The role of cytokines as inflammatory mediators in preeclampsia. Pan Afr Med J 2015; 20:219

5 Kah M, Levy L, Brown C. Potential for effects of land contamination on human health. 1. The case of cadmium. J Toxicol Environ Health B Crit Rev 2012;15(05):348-363

6 Thévenod F, Lee WK. Toxicology of Cadmium and Its Damage to Mammalian Organs. Met Ions Life Sci 2013;11:415-490

7 Al-Bader A, Omu AE, Dashti H. Chronic cadmium toxicity to sperm of heavy cigarette smokers: immunomodulation by zinc. Arch Androl 1999;43(02):135-140

8 Hamden K, Carreau S, Marki FA, Masmoudi H, El Feki A. Positive effects of green tea on hepatic dysfunction, lipid peroxidation and antioxidant defence depletion induced by cadmium. Biol Res 2008;41(03):331-339

9 Nishijo M, Satarug S, Honda R, Tsuritani I, Aoshima K. The gender differences in health effects of environmental cadmium exposure and potential mechanisms. Mol Cell Biochem 2004; 255(1-2):87-92

$10 \mathrm{Hu}$ KH, Li WX, Sun MY, et al. Cadmium induced apoptosis in MG63 cells by increasing ROS, activation of p38 MAPK and inhibition of ERK 1/2 pathways. Cell Physiol Biochem 2015;36(02):642-654

11 Kolusari A, Kurdoglu M, Yildizhan R, et al. Catalase activity, serum trace element and heavy metal concentrations, and vitamin A, D and E levels in pre-eclampsia. J Int Med Res 2008;36(06):1335-1341

12 Schroeder HA, Buckman J. Cadmium hypertension. Its reversal in rats by a zinc chelate. Arch Environ Health 1967;14(05):693-697

13 Wang F, Fan F, Wang L, Ye W, Zhang Q Xie S. Maternal cadmium levels during pregnancy and the relationship with preeclampsia and fetal biometric parameters. Biol Trace Elem Res 2018;186 (02):322-329

14 Zhang Q Huang Y, Zhang K, et al. Progesterone attenuates hypertension and autoantibody levels to the angiotensin II type 1 receptor in response to elevated cadmium during pregnancy. Placenta 2018;62:16-24

15 Bara L, Bara C, Bara V, et al. Report on the influence of heavy metals on the evolution of the pregnancy in smoking mothers. 2010

16 Lin F, Zeng P, Xu Z, et al. Treatment of Lipoxin A(4) and its analogue on low-dose endotoxin induced preeclampsia in rat and possible mechanisms. Reprod Toxicol 2012;34(04):677-685

$17 \mathrm{Xu} \mathrm{Z}$, Zhao F, Lin F, et al. Preeclampsia is associated with a deficiency of lipoxin A4, an endogenous anti-inflammatory mediator. Fertil Steril 2014;102(01):282-290.e4 
e208 The Effect of BML-111 in Preeclampsia Rat Model Zhang et al.

18 Zhang Q, Huang Y, Zhang K, et al. Cadmium-induced immune abnormality is a key pathogenic event in human and rat models of preeclampsia. Environ Pollut 2016;218:770-782

19 Kukongviriyapan U, Apaijit K, Kukongviriyapan V. Oxidative stress and cardiovascular dysfunction associated with cadmium exposure: beneficial effects of curcumin and tetrahydrocurcumin. Tohoku J Exp Med 2016;239(01):25-38

20 Houston MC. The role of mercury and cadmium heavy metals in vascular disease, hypertension, coronary heart disease, and myocardial infarction. Altern Ther Health Med 2007;13(02):S128-S133

21 Olszowski T, Baranowska-Bosiacka I, Gutowska I, Chlubek D. Proinflammatory properties of cadmium. Acta Biochim Pol 2012;59 (04):475-482
22 Faroon O, Ashizawa A, Wright S, et al. Toxicological Profile for Cadmium. Atlanta, GA: Agency for Toxic Substances and Disease Registry; 2012

23 Chan HM, Cherian MG. Mobilization of hepatic cadmium in pregnant rats. Toxicol Appl Pharmacol 1993;120(02):308-314

24 Szabo S, Mody M, Romero R, et al. Activation of villous trophoblastic p38 and ERK1/2 signaling pathways in preterm preeclampsia and HELLP syndrome. Pathol Oncol Res 2015;21(03): 659-668

25 Sharma A, Satyam A, Sharma JB. Leptin, IL-10 and inflammatory markers (TNF- $\alpha$, IL-6 and IL-8) in pre-eclamptic, normotensive pregnant and healthy non-pregnant women. Am J Reprod Immunol 2007;58(01):21-30 